\title{
The future of digital platforms: conditions of platform overthrow
}

Maxime, Thomas ${ }^{1}$, MINES ParisTech, PSL University, Centre de gestion scientifique (CGS), i3 UMR CNRS, 60 Bd St Michel 75006 Paris, France

Pascal Le Masson, MINES ParisTech, PSL University, Centre de gestion scientifique (CGS), i3 UMR CNRS, 60 Bd St Michel 75006 Paris, France

Benoit, Weil, MINES ParisTech, PSL University, Centre de gestion scientifique (CGS), i3 UMR CNRS, 60 Bd St Michel 75006 Paris, France

Julien, Legrand, MINES ParisTech, PSL University, Centre de gestion scientifique (CGS), i3 UMR CNRS, 60 Bd St Michel 75006 Paris, France

\begin{abstract}
Are the digital platforms that we know here to stay? Both empirical insights and theoretical works suggest digital platform stability. Digital platforms such as Airbnb, Netflix and Taobao have experienced tremendous success, and the theoretical works on modularity and multisided markets depict competitive platform landscapes as controlled by a hegemonic platform leader. However, platform history chronicles multiple cases of leadership shifts in platform ecosystems. In this paper, we coin these situations "platform overthrows" and uncover the relevant strategies for both a challenger to conduct a platform overthrow and for a platform leader to resist it. To do so we conducted an inductive protocol to re-interpret 27 already-published cases of platform overthrow attempts. Our results suggest that, during a platform overthrow attempt, both players articulate functional expansion and technical genericity. Accordingly, we formulate propositions that account for the strategies at play during a platform overthrow attempt. We discuss these results regarding the digital platform literature on competition and conclude that the study of platform overthrow can yield useful insights on the future of digital platforms.
\end{abstract}

Keywords: Digital Platforms, Platform Overthrow, Competition, Double sided-market, Strategy

\footnotetext{
${ }^{1}$ Corresponding author
} 


\section{1) Introduction: Are digital platforms here to stay?}

Recent years have witnessed the rise of several iconic digital platforms, e.g. Airbnb, Uber, Netflix, BlaBlaCar and Taobao, that have boldly challenged incumbent companies and established themselves as market leaders in only a few years' time. These new players have not only changed the way that we find accommodation, take a cab, watch movies, and shop but also conquered what seem to be unchallengeable market positions. As their successes are now sufficiently clear, researchers are left with the remaining question of their lifecycle (Muzellec, Ronteau, and Lambkin 2015) and the nature of competition in a digital platform world (Kazan et al. 2018). What can research say about the future of digital platforms? Are digital platforms here to stay (de Reuver, Sørensen, and Basole 2018)? Or are there strategies to overthrow an established platform leader?

As noted by the literature, the swift success of digital platforms is rooted in their ability to harness the logic of the platform revolution (Parker, Van Alstyne, and Choudary 2016). These companies succeeded not because they are digital but because they implemented a platform strategy by using digital assets and relevant technologies to become platform leaders (Gawer and Cusumano 2002); firms that orchestrate an innovation ecosystem where multiple complementors develop complementary innovations. When investigating the future of digital platforms, one may be able to examine the platform literature for insights. On the one hand, this literature anticipates a stable competitive landscape: thanks to modularity (Baldwin and Clark 2006; Baldwin and Woodard 2009) and network effects at play in multi-sided market (Rochet and Tirole 2003), platform leaders are bound to continually reinforce their leadership on a given innovation ecosystem. On the other hand, empirical studies underline that platform ecosystems are more unstable than what is indicated in these straightforward theoretical approaches. The few empirical studies that have conducted largescale, cross-industry analyses on platform ecosystems report on the high rate of failure even for established platform leaders (Yoffie, Gawer, and Cusumano 2019; Reeves et al. 2019). Industry-specific studies also report on rapid platform leadership shifts such as in video games (Gallagher and Park 2002) or mobile phone (Kenney and Pon 2011) ecosystems. Finally, the industry platform literature recognizes that complementors can innovate to compete with the platform leader (Gawer 2014). Therefore, empirical analysis seems to indicate that a competitive platform landscape is unstable and submits to rapid changes in platform leadership.

In this paper, we investigate the functional and technical innovation strategies at play during an attempt of platform overthrow. We define "platform overthrow" as situations in which a platform leader loses its architectural control of the platform for the profit of a challenger that, initially, is strategically weaker. As we shall see, platform overthrow is a phenomenon that has received scant attention from the literature and the available strategies for these situations remain rather fuzzy. Accordingly, we conduct an inductive research protocol to uncover the type of functional and technical innovations that can lead to platform overthrow. To do so, we rely on a rich secondary material composed of 27 published descriptions of attempt of platform overthrow, almost all academically-related. These data suggest that a challenger can overthrow a platform by articulating functional and technical innovations. By conducting an inductive research protocol based on the 27 cases, we were able to qualify the type of functional and technical innovations at stake during an attempt of platform overthrow. Our results suggest that both platform leader and challenger (i) engage in functional expansion that is to say introduce new functions that are neither an optimization nor a combination of existing functions and that they (ii) try to increase the genericity of their technical core. Put differently, they change their technical core in order to increase its capacity to address many existing and emerging functions and create value across a broad range of applications. Because of the richness of our secondary research material, we were also able to relate functional expansion and technical genericity with platform overthrow outcome. As we shall see, by articulating functional expansion and technical genericity, a challenger can overthrow an established platform and, conversely, an established platform leader can resist an overthrow attempt. We discuss the implications of these results for digital platform strategy and conclude on the insights that functional expansion and technical genericity bring to foresee the future of digital platforms.

The rest of the paper is structured as follows. First, in the literature review, we show that scientific platform models overemphasize platform stability despite empirical examples of successful platform overthrow. We examine the literature for weakening mechanisms that competitors could leverage in a platform overthrow attempt and formulate our research questions on the type of functional and technical innovations at play during an attempt of platform overthrow. Second, in the methodology section, we detail how we draw from classical inductive methodology to analyze our secondary material that describes platform overthrow attempt and how the richness of this material enable us to conduct a statistical test that strengthens our results. Third, in the results section, we present both our inductive and statistical results that suggest that the articulation of functional expansion and technical genericity by a challenger can lead to platform overthrow. Fourth, in the discussion section, we show that the notions of functional expansion and technical genericity are coherent with the existing literature and we discuss their implications for the research on platform competition and the future of digital platforms. 


\section{2) Literature review: The lack of research on the functional and technical innovations at play during an attempt of platform overthrow}

Platforms and platform leaders have become major subjects in academic fields over the past few years. In this section, we build on this constant academic progress to identify the issues of platform dynamics and competition with a two-step approach. First, we point out that empirical research reports on leadership shifts in platform ecosystems, while the classic scientific models - modularity and the multisided market - do not account for them. Second, we call these shifts "platform overthrows" and examine the literature for innovation strategies that lead to platform overthrow. We take note of the lack of research on the functional and technical innovations at play during an attempt of platform overthrow and formulate our research questions accordingly.

\subsection{Highlighting a discrepancy in the literature on platform dynamics: platform models emphasize stability, while empirical studies report on platform overthrow situations.}

Early academic work on the platform industry was initiated at the end of the 90 s to explain the success of several firms in the software sector that had achieved rapid market domination (Steinberg 2017). Industry platforms are described as "products, services, or technologies developed by one or more firms, which serve as foundations upon which a large number of firms can build further complementary innovations" (Gawer and Cusumano 2014). Industry platforms are thus specific innovation ecosystems in which platform leaders - one or several firms - orchestrate innovation through a specific technical core (Thomas, Autio, and Gann 2014). Plentiful empirical evidence from the technological management literature has described how companies such as Intel, Palm or DoCoMo, which are acknowledged as platform leaders, relied heavily on complementary innovations to ensure their market success (Gawer and Cusumano 2002). More recently, the literature highlight that, beyond famous platform leaders (Geissinger, Laurell, and Sandström 2020), platform-related logics are implemented by smaller actors in sectors such as the sharing economy (Sanasi et al. 2020), healthcare (Fürstenau et al. 2019), or fashion (Schmidt et al. 2020).

Two main bodies of theoretical works, from both the engineering design and industrial economic literature, have modeled the underlying mechanisms that a platform leader uses to foster efficient complementary innovation. On the one hand, the modularity literature explains how platform leaders promote complementary innovations (Baldwin and Clark 2006). Because of modular systems, some parts can be designed by "complementors" that are in charge of providing bestin-class modules (K. Boudreau 2012; Baldwin and Woodard 2009), while the platform leader is responsible for the coherence of the overall structure and the modules' compatibility (Gawer and Cusumano 2014; Thomas, Autio, and Gann 2014). Modularity is key for the platform leader as it is a condition to nurture a thriving innovation ecosystem (Hatchuel, Masson, and Weil 2009). On the other hand, the multisided market literature provides the theoretical prism to understand how to achieve rapid market domination with complementary innovation (Rochet and Tirole 2003). The multisided market approach assumes the existence of at least two groups of heterogeneous platform users that are subjected to indirect network effects, which means that the different sides of a platform can mutually benefit from the size and characteristics of the other side. When more customers from one side join the platform, the other side is more interested in joining, which leads to a virtuous circle. A critical issue for a multisided market is to solve the "chicken and egg problem"; however, once a critical mass in the installed base of customers is reached, it becomes difficult for others to compete with the platform (Evans 2009). Therefore, a platform leader should develop complementary innovation to ensure that both sides join and stay on board. Thus, by combining modularity and multisided network effects, the academic literature provides good insights into the dynamics at stake that enable the rise of a successful platform leader and that anticipate the platform's long-run market dominance. Both engineering design frameworks (modularity) and economic framework (multi sided market) anticipate stability in platform dynamics.

These straightforward models of platform dynamics indicate that a platform leader, once established, remains in control of a thriving innovative ecosystem. However, these assumptions have been undermined by recent studies on platform ecosystem evolutions. Thus, based on empirical observations, several scholars point out that industry platforms are dynamic structures that experience different stages over time (Gawer 2014; Thomas, Autio, and Gann 2014; Muzellec, Ronteau, and Lambkin 2015; Hein et al. 2019). They also describe that a platform ecosystem can be damaged to a point where the platform leader can no longer orchestrate innovation (Yoffie, Gawer, and Cusumano 2019; Reeves et al. 2019). As if this prospect was not worrisome enough for platform leaders, the literature accumulates examples of platform leaders that lose their architectural control for the profit of a complementor (Legrand et al. 2017): for example, IBM losing its architectural control over the PC platform for the profit of Intel (Gawer and Cusumano 2002), Nokia facing the rise of Microsoft OS and losing control over the Symbian platform (Kenney and Pon 2011) and Yahoo! becoming Google's 
complementor (Rindova et al. 2012). In these examples, the platform leaders were widely recognized for their ecosystem orchestration capabilities; therefore, the most disturbing fact is that they fall not because of an ecosystem orchestration mistake but due to the successful strategy of a competitor. In this article, we term these situations as "platform overthrow" and define them as situations in which platform leaders lose their architectural control over the platform for the profit of a challenger that, initially, is strategically weaker.

When comparing theoretical and empirical approaches on platform fall, one can note a discrepancy between platform models that emphasize stability and empirical studies that report on platform overthrow situations. This is identified in the literature as a relevant gap linked with platform dynamics issues. As noted by a recent literature review (McIntyre and Srinivasan 2017): "There is relatively little understanding of platform dynamics and their evolution, with platforms being treated as systems that remain relatively stable overtime [...] As a result, additional research is needed that focuses on how platform firms manage and leverage their portfolio of complements during regimes of frequent technological change". Thus, understanding the strategies at play during an attempt of platform overthrow appears as a relevant gap in the literature.

\subsection{Fuzziness of innovation strategies that weaken an established platform leader}

The literature reports on several mechanisms, both external and internal, that can weaken an established platform leader. External mechanisms include change of legislation (Bazarhanova, Yli-Huumo, and Smolander 2019), statesupported competitor (Cusumano, Gawer, and Yoffie 2019) or worldwide pandemic (Rani and Dhir 2020) while internal mechanisms include lack of trust in the network (Gawer and Henderson 2007), poorly designed pricing system (K. J. Boudreau and Jeppesen 2015) or lack of leadership (Reeves et al. 2019). Each of the previous mechanisms could be leveraged by a challenger as a strategy to overcome an installed platform leader.

In this paper, we focus only on strategies linked with functional innovation and technical innovation. As we shall see, the literature acknowledges that both functionally-based and technically-based innovations can weaken an established platform leader. However, the scant description of the nature of these innovations brings ambiguity on how a challenger could leverage functional and technical innovations up to the point of overthrowing an established platform leader.

\subsubsection{Functional innovation}

Functional innovation is identified in the literature as a mechanism that weakens a platform leader. Empirical evidence shows that platform leaders are subjected to the innovators' dilemma (Gawer and Cusumano 2014): thus several platform leaders were challenged because they neglected the importance of new functions (Suarez and Kirtley 2012). However, as these studies fail to characterize the exact nature of the functional innovations that weaken an established platform leader, they provide little guidance for a challenger that aims at overthrowing an installed platform. They only precise that new functions have to be "coherent" with technical aspects (Gawer and Cusumano 2014) and should be carefully designed to allow "differentiation" (Suarez and Kirtley 2012).

To the best of our knowledge, the only work that identifies a type of functional innovation that can lead to platform overthrow is the one conducted on envelopment strategy (Eisenmann, Parker, and Van Alstyne 2011). Envelopment strategy refers to a situation in which a platform A is able to propose for free, in top of its current offer, the functions of a platform B. This strategic move decreases the switching costs for consumers who desert platform B leading to its fall. The theoretical work on platform envelopment therefore identifies one specific functional innovation including all the functions of the targeted platform leader in one's own offer for free - that can be used to overthrow a platform. However, platform envelopment is a very demanding strategy: all the functions have to be bundled for free. Therefore, one could wonder if envelopment is the only type of functional innovation that can lead to platform overthrow.

Thus, functional innovation is identified as a mechanism that can weaken an established platform leader. However, except for the demanding platform envelopment strategy, the literature remains relatively unclear concerning the nature of the functional innovations that a challenger can leverage to overthrow an established platform leader.

\subsubsection{Technical innovation}

Technical innovation has for long been recognized as a strategic game changer in innovation ecosystems (Garud and Kumaraswamy 1993; Baldwin and Woodard 2009) and the literature accumulates empirical examples that show that specific technical innovations can weakened a platform leader (Gallagher and Park 2002; Kenney and Pon 2011; West and Wood 2013; Den Hartigh et al. 2016). However, in the platform literature, theoretical approaches are prone to describe technical innovation as a mechanism that strengthens platform leaders' positions (Baldwin and Woodard 2007; Hatchuel, 
Masson, and Weil 2009; Thomas, Autio, and Gann 2014; Kokshagina et al. 2016). Therefore, the literature gives only scant indications concerning how a challenger can leverage technical innovations to overthrow an established platform.

To the best of our knowledge, only two studies provide frameworks accounting for the technical innovations that can overthrow a platform leader. The first one explains how a challenger can endanger an established platform leader that poorly manages technical transitions (Ozalp, Cennamo, and Gawer 2018). First, this study shows that, by introducing a new technology that is too demanding in terms of learning for complementors, an established platform leader can disrupt its own ecosystem. Second, the study shows that, by designing a technology that reduces the cost for the complementors to learn about and develop for its own platform core, a challenger can overthrow the platform leader. Therefore, this study identifies a specific type of technical innovation - a technical core that reduce the learning cost for complementors - that could lead to overthrow. However, it is worth noting that this strategy is triggered by an initial mistake of the established platform leader that fails to provide the right balance between learning costs for complementors and technical advances of its platform core. The second piece of research that identifies a specific technical innovation that could be leveraged to overthrow a platform is related to the issue of platform openness (Karhu and Ritala 2020). It accounts for cases in which the challenger "makes an exact copy of all boundary resources upon which complements depend (e.g., APIs) such that, from the complementor's perspective, the platform looks exactly the same as the incumbent's platform" (Karhu and Ritala 2020, p.8). In such cases, the marginal cost of complementors to switch is very low, resulting in a deadly threat to the established platform leader. However, the study underlines that the related technical innovations can only be designed if the established platform leader opens its technical core too widely to enable copying.

Empirically, technical innovation is identified as a mechanism that can weaken an established platform leader. However, the literature only gives scant indications on how a challenger could leverage technical innovation to overthrow a platform leader as the existing insights are limited to specific situations in which the established platform leader makes mistakes.

\subsection{Research question: what are the functional and technical innovation strategies at play during an attempt of platform overthrow?}

By documenting the lack of theoretical explanations for platform overthrow and the lack of guidance for a challenger willing to leverage functional and technical innovations to overthrow an established platform leader, our literature review shows that the innovation strategies at play during an attempt of platform overthrow represent a legitimate exploratory research question.

Therefore, in this article, we address the following research questions. What are the functional and technical innovation strategies at play during an attempt of platform overthrow? How does a challenger articulate functional and technical innovations to take over the architectural control of a platform ecosystem at the expense of its former platform leader? How, reciprocally, does a platform leader faced with an attempt of platform overthrow articulate functional and technical innovations to maintain its architectural control over its platform ecosystem?

\section{3) Methodology: an inductive protocol based on 27 descriptions of attempt of platform overthrow}

Given the lack of previous studies on platform overthrow and the exploratory nature of our research questions, we decided to conduct an inductive research protocol. However, in a socio-economic world that celebrates platform stability, a platform overthrow attempt is rarely seen as such by the actors involved in these competitive moves. Therefore, an interview-based inductive protocol seemed (i) complicate to set up due to the difficulty to identify platform overthrow attempt situations and (ii) complicate to analyze due to unforeseen and uncontrollable biases that actors may have on the subject of platform overthrow. Fortunately, empirical research describing platform history that has been conducted for the last twenty years provides an interesting research material to investigate our research question because, as we shall see, in this material (i) platform overthrow attempts are relatively easy to identify and (ii) the existing biases can be mitigated. Therefore, we decided to conduct an inductive research protocol based on secondary data.

In this methodology section, we explain how we adapted the classical inductive methodology (Gioia, Corley, and Hamilton 2012) to the study of secondary data. We first present how we collected 27 descriptions of platform overthrow attempt. Second, we explain how we analyzed the collected materials. Then, we highlight how the richness of our secondary research materials enabled us to conduct a basic statistical test that strengthens our results. 


\subsection{An exhaustive attempt to gather secondary data describing the evolution of functional and technical innovations during an attempt of platform overthrow}

In this sub-section, we present how we collected a sample of 27 descriptions of platform overthrow attempt.

In an inductive protocol, the representativeness of the cases is critical. However, given the lack of research on platform overthrow, we were confronted to the impossibility of relying on an ex-ante framework that could guide us through the case selection process. Therefore, our strategy was first to make an exhaustive attempt to collect a rich secondary material on platform overthrow attempt and then to assess that the sample collected displays a minimum level of representativeness.

To find rich descriptions of platform overthrow attempt, we relied on two sources: academic papers and books that account for long term platform history. To find relevant academic papers, two search strategies were simultaneously implemented in Google Scholar: a general search strategy on the topic of platform competition (with entries such as "platform competition," "platform disruption," or "market entry in platform ecosystem") and a specific search strategy focusing on famous platform battles (with entries such as "Netscape versus Microsoft," "Cisco competitors," or "Steam platform history"). These two search strategies yielded hundreds of papers. The vast majority of them were disregarded based on criteria that could be assessed swiftly: the description of the platform overthrow attempt is anecdotal in the paper (Eisenmann, Parker, and Van Alstyne 2011), the paper does not mobilize platform as a theoretical construct (Kay 2013), the description of the platform overthrow attempt is only used to exemplify a theoretical construct and therefore the description is limited (Cennamo and Santalo 2013). This process allowed us to gather fifty-five academic articles presenting an empirical description of a competitive outbreak faced by a platform leader. When selecting books out of all the ones that are written on platforms, we favored academic-related books (Cusumano and Selby 1988; Gawer and Cusumano 2002; Evans, Hagiu, and Schmalensee 2006; Cusumano, Gawer, and Yoffie 2019). We also added two practitioners' books in our sample : one because it complemented an academic description (Bunnell and Brate 2000) and the second because Netflix is a relatively young platform whose history is only accounted for by journalistic-related materials (Keating 2013).

We then assessed the relevance of these books and academic papers for our inductive research protocol by considering two questions: (1) Can the description made in the material be considered as an attempt of platform overthrow? (2) Does the description account for functional and technical innovations that occur during the competitive struggle? To qualify as research material, a description should present a positive answer to these two questions. To assess the first question, we looked for unambiguous statements in the material testifying that an attempt of platform overthrow is underway. For the second question, two of the four co-authors red each paper, discuss it, and then came to an agreement of whether the description account for functional and technical innovations. At the end of this process, we were able to collect 27 descriptions of attempt of platform overthrow: seven coming from five academic papers (Gallagher and Park 2002; West and Wood 2013; Staykova and Damsgaard 2015; Den Hartigh et al. 2016; Glimstedt 2020) and twenty-two coming from the aforementioned books. For illustrative purposes, Table 1 elucidates some of these descriptions, their sources and the quote that make them belong to our research material. For each mentioned source, the number of cases is also indicated.

We then verified that the research material collected displays a minimum level of representativeness. Platform literature identifies several research biases in the field such as focusing only on successful platform leaders (de Reuver, Sørensen, and Basole 2018), conducting studies only in a single industry setting (de Reuver, Sørensen, and Basole 2018), and separating the economic and the engineering design theoretical constructs (Gawer 2014). This last bias is known to overemphasize the separation between transaction platforms - defined as intermediaries or online market places - and innovation platforms - defined as technological building blocks upon which complementary innovations can be developed - while leading companies are nowadays hybrids as they articulate both approaches (Cusumano, Gawer, and Yoffie 2019). As we wanted to make sure that our research material mitigates these three biases, we assessed, for each description, the competitive outcome, the industrial setting and the initial theoretical lenses used to analyse the platform overthrow attempt.

Our research material avoids the success bias: there are fifteen descriptions of successful attempt of platform overthrow (the established platform leader is overthrown) and twelve descriptions of failed attempt (the established platform leader remains in control of the ecosystem). Our research material also mitigates the industry setting bias: even if a significant number of cases come from the personal computer industry - eight cases - and from the mobile industry - seven cases - other sectors are also represented such as the telecommunication infrastructure industry, the online selling 
industry, the film industry, the video game industry and the banking industry. Last but not least, our material limits the bias related to the frameworks used to describe the overthrow attempts: twelve descriptions were mainly conducted with economic constructs (multi-sided market), twelve were mainly conducted with engineering design constructs (modularity) and five were conducted with constructs from both research traditions. Our research material is slightly unbalanced concerning platform type: eighteen attempts relate to innovation platform; six attempts relate to transaction platform and three relate to hybrid companies. Table 2 elucidates our research material of 27 cases of platform overthrow attempt, each case is given a number so that we can refer to it in the results section.

Insert Table 2 here

\subsection{Adapting classical inductive protocol to the analysis of secondary data}

To analyze our data, we followed the classical recommendations for inductive research protocol (Gioia, Corley, and Hamilton 2012). More precisely, we conducted two similar inductive protocols: the first one to identify the type of functional innovations at stake during an attempt of platform overthrow and the second to identify the type of technical innovations at stake during an attempt of platform overthrow. This resulted in two Gioia's diagrams that are presented in the results section.

At this point, we shall elucidate the influence of biases induced by the use of secondary data in our inductive protocol. When using secondary data, one may adhere to the interpretation biases introduced by the initial authors. In our case, the existence of such biases is not a problem as they strengthen our research protocol. Because platform research historically highlights platform stability, scholars describing platform competition are probably biased toward stability. Therefore, when describing a platform overthrow, they are probably prone to explain the fall of a platform leader with reasons that are exogenous of the competitive battle (a mistake from the platform leader, a change in legislation...). Therefore, if we find evidence in the secondary material that functional and technical innovations can lead to platform overthrow, these evidences are probably underestimated which strengthens our future results that aim at showing that there are functional and technical innovations that can overthrow an established platform leader.

The inductive protocol conducted is the following. Two of the authors red all the descriptions and gather all the quotes referring to functional and technical innovations in each case. We considered the quotes as our $1^{\text {st }}$ order concepts because they are a first conceptual work conducted by the initial authors. To generate $2^{\text {nd }}$ order themes, the quotes were then shared with the other co-authors during several meetings so as to group them and label them. The research material and the inductive analysis conducted were then presented to other researchers in order to get feedbacks that led to the modification of several $2^{\text {nd }}$ order themes. We then draw from our own knowledge of the engineering design and industrial economic literatures to propose relevant aggregate dimensions.

\subsection{A statistical test, allowed by the richness of our secondary material, that strengthens the results of our inductive protocol}

The richness of our secondary data allowed us to conduct a basic statistical test that strengthens the results generated by our inductive protocols. This section presents the logical ground on which this test was conducted.

First, we shall underline that our research protocol generates two variables: (i) the outcome of 27 platform overthrow attempts (who is the player who won the competitive struggle?) and (ii) the types of functional and technical innovations conducted during an attempt of platform overthrow. If the types of innovation identified by the inductive protocols are relevant strategies in situation of platform overthrow attempt, then the player who performed these innovations would have a competitive advantage over the other. There would therefore be a link between the player who conducts these innovations and the outcome of the attempt. Conversely, if the types of innovation identified are not relevant strategies in situation of platform overthrow, then the two variables would be independent: whether or not the platform leader (or the challenger) performs these innovations would have no link with the outcome.

Therefore, by testing the dependency between the outcome of an attempt and the player who performs the types of innovations identified, we shall be able to see if the type of innovation identified are relevant strategies during a platform overthrow attempt. To test this dependency, we conducted a $\chi^{2}$ with a $0.1 \%$ confidence interval (for further details on the test conducted, please see subsection 4.3 as some aspects can only be discussed once the innovation types are identified). 


\section{4) Results: Functional expansion and technical genericity as the type of functional and technical innovations conducted during an attempt of platform overthrow}

Our research material yields interesting results concerning the type of both functional and technical innovations conducted during an attempt of platform overthrow. From a functional point of view, our data suggest that both challengers and platform leaders solve long lasting functional issues, add new functions on top of existing ones and try to increase the potential of the ecosystem. By conducting these three types of functional innovation, the players engage in what we call a functional expansion. From a technical point of view, our data suggest that both players articulate technical core reconfiguration, technical core transition and technical core change of boundaries in order to cope with functional expansion. These three strategic moves aim at increasing what we call the genericity of the technical core.

\subsection{Functional expansion: characterizing the type of functional innovation at play during an attempt of platform overthrow}

Challengers face the architectural power of the platform they want to overthrow. An installed platform is a dynamic player that constantly sharpens its offers and optimizes the existing functions it proposes. How do challengers leverage functional innovation to overthrow a platform leader? Conversely, what kind of functional innovations a platform leader can design when confronting a fierce attempt of overthrow? Our data suggest that players try to (i) solve long lasting functional issues, (ii) add new functions on top of existing ones and (iii) increase the potential of the ecosystem.

As an example of how a challenger tries to solve long lasting functional issues faced by the platform leader, consider case 2 and the battle between IBM and Intel. To compete with the dominant PC architecture, Intel engineers developed a chip, the PCI, that solved problems of the PC architecture "We picked [Intel's PCI] because it solved a range of problems: It solved a graphics problem, it solved plug and play problems, it solved interrupt problems, it solved performance issues" (Gawer et Cusumano, 2002, p.27)

To compete with established platform leaders, some challengers both solve long lasting functional issues and add new functions on top of existing ones as in case 18 where Firefox challenged the existing internet browser platform Internet Explorer. Our data report that the initial aim of Mozilla developers was to solve the functional issues of the incumbent platform: "Seeking to avoid software bloat and performance issues, two developers working for the Mozilla nonprofit foundation began what would become the Firefox browser in late 2002. Their goal was to produce a standalone browser that would be faster, simpler, and more secure" (Cusumano, Gawer, Yoffie, 2019, p.72). However, to seriously compete with Internet Explorer, the developers also added new functions on top of existing ones: "Mozilla introduced its first browser in June 2002, with innovative features such as tabbed browsing, the ability to select a word or phrase and search the web for the selected text, and the ability to store common information for automatically filling in forms" (Cusumano, Gawer, Yoffie, 2019, p.72).

Some functional innovations are not just an addition of a new functionalities but rather seek to increase the potential of the ecosystem. Thus, Case 17 reports how Alibaba, with its Taobao website, engaged in a strategy to renew the functions proposed at the time by eBay and therefore competed with what was the incumbent platform leader in China. "Taobao's first web pages were copied directly from eBay's design. But Alibaba quickly pivoted, recognizing that one cannot win a platform battle with a me-too product (Cusumano, Gawer, Yoffie, 2019, p.69-70). New functions were introduced to increase the potential of the ecosystem: "While eBay initially did not let buyers and sellers interact directly [...], Taobao encouraged buyers and sellers to communicate with each other and added instant messaging to the website, allowing buyers and sellers to build trust" (Cusumano, Gawer, Yoffie, 2019, p.70). Other functions were added to build trust among Chinese customers that do not own a credit card: "Alibaba launched its own online payment system called Alipay, similar to PayPal, which eBay had acquired shortly before entering the Chinese market. Alipay, unlike PayPal, used an escrow model. At the time of a transaction, funds would go into an escrow account and would only be released after the buyer had received and inspected the item." (Cusumano, Gawer, Yoffie, 2019, p.70). Because it introduced functions that increased the potential of the ecosystem, Alibaba surpassed eBay and became the leading platform in China.

Solving long lasting functional issues, adding new functions on top of existing ones and increasing the potential of the ecosystem are also weapons an incumbent platform leader can use to resist an attempt of platform overthrow. As an example of how a platform leader can add new functions on top of existing ones and increase the potential of the platform ecosystem, consider case 14 and the battle between Symbian and Microsoft: to compete with the platform Microsoft was trying to build, Symbian partners developed mobile phones that displayed new function features for both the customer and the provider side. On the customer side, Symbian added new functions on top of existing ones: "Beyond the ability to make voice calls on GSM mobile phone networks, the phones inherited the capabilities of Psion's organizers 
(such as a calendar and address book) to which Symbian and its partners added features suitable for a mobile Internet device (such as e-mail and web browsing)" (West and Wood, 2013, p.33). On the supplier side, Symbian increased the potential of the ecosystem by transforming the closed platform a mobile was at the time into "a cellular phone that was also a programmable mobile computing device" (West and Wood, 2013, p.29) that offered software developers the functionalities they were encountering on a personal computer system.

When considering that both players try to solve long lasting functional issues, to add new functions on top of existing ones and to increase the potential of the ecosystem, one can note that the functional innovations introduced during an attempt of platform overthrow are neither an optimization nor a combination of existing functions. Both the challenger and the established platform leader rely on a form of radical functional innovation. To highlight this radicalness, we suggest to qualify the functional innovation performed by the players during an attempt of platform overthrow as a functional expansion.

Functional expansion is a notion that originates from the industrial economic literature (El Qaoumi et al. 2018). A functional expansion is conceptualized as a functional innovation that is neither an optimization, nor a combination of existing functions. To us, this notion accounts for the functionally-related design efforts undertaken during an attempt of platform overthrow. Figure 1 sums up the inductive logic that led us to that conclusion. We illustrate through this figure the analysis aforementioned and account for all the cases in which quotes were found that relate either to solving long lasting functional issues, to adding new functions on top of existing ones or to increasing the potential of the ecosystem.

Insert Figure 1 here

\subsection{Technical core genericity: characterizing the type of technical innovation at play during an attempt of platform overthrow}

Our data suggest that functional expansion cannot on its own account for the innovations that take place during an attempt of platform overthrow as the players also conduct technical innovations. How do challengers leverage technical innovations to overthrow a platform leader? Conversely, what kind of technical innovations a platform leader can design when confronting a fierce attempt of overthrow? Our data suggest that during an attempt of platform overthrow, players articulate (i) technical core reconfiguration, (ii) technical core transition and (iii) technical core change of boundaries.

Our data suggest that a platform leader can resist an attempt of platform overthrow by reconfiguring its technical core. Case 4 is an emblematic example that shows how Microsoft reconfigured Windows to react to the competitive threat that Netscape represented with its Navigator browser. The Netscape browser was a serious threat to Microsoft as it added new functions on top of existing ones: "customers can launch software applications from Web servers that interact with Internet browsers, rather than with Windows, making the browser a competing platform" (Gawer and Cusumano, 2002, p.93) The reaction of Microsoft was swift: "Microsoft executives and senior engineers quickly decided to embed the browser in Windows 95 and head off this threat. [...] Microsoft [...] built its own browser, Internet Explorer, to add to Windows" (Gawer and Cusumano, 2002, p.145). The reaction of Microsoft was clearly a technical reconfiguration as "Microsoft took on the challenge [...] bundling a browser with Windows" (Gawer and Cusumano, 2002, p.154). Our data show that the reconfiguration of the technical core can also take place after the established platform leader has bought its challenger: case 6 shows that while Cisco bought its challenger Stratacom and therefore reduced the threat, it also reconfigured its technology to include the functions that had allowed Stratacom to thrive. Cisco, as a provider of network infrastructures, feared Stratacom that, through new switches, had introduced a function that had increased the potential of the ecosystem: "ATM switches [that] could connect traditional phone networks to modern data networks" (Gawer and Cusumano, 2002, p.173). Therefore, Cisco bought Stratacom but also "redefined its Internet networking platform as containing either or both non-ATM routers and ATM technologies" (Gawer and Cusumano, 2002, p.174). This change was conducted at a technical level as "bits and pieces of the IOS ran through and connected Cisco technology [...] One of the first things Cisco did with the technology from acquisitions - StrataCom's switches, for example-was to add IOS code to its platform so that it could work with Cisco's existing product lines" (Bunnell, 2000, p.118). Thanks to its strategy, Cisco resisted Stratacom's overthrow attempt. Therefore, our data suggest that in a situation of platform overthrow attempt, classic reactions to a competitive threat - such as buying the challenger - is coupled with a reconfiguration of the technical core of the platform leader.

Our data indicate that a challenger can overthrow a platform leader by conducting a technical core transition and, once established, resist subsequent attempts of overthrow with technical core reconfiguration. That is what Sony did with the PlayStation in case 12: Sony first overthrew Nintendo and its Super Nintendo Entertainment System (NES) by proposing an advanced technical core - the PlayStation - and then resisted the introduction of the N64 (that was a 
technically improved platform compared to Super NES) by making the most of its newly designed technical core. Thus, Sony "introduced the first commercially successful machine with an operating system and with applications that came on a CD rather than on a chip" (Evans, Hagiu, and Schmalensee, 2006, p.129) while Nintendo "decided to continue using cartridges, both for the Super NES and for the N64" (Evans, Hagiu, and Schmalensee, 2006, p.130). Betting on CDROMs was a way to cope with the functional expansion of this sector - game releases - as it allowed "a significant improvement in game complexity and [easy access] if game makers needed to increase production" (Evans, Hagiu, and Schmalensee, 2006, p.130). Once established, the PlayStation became a platform that Sony could reconfigure to resist the introduction of the N64: "the software platform for the Playstation was a proprietary Sony operating system developed in-house [and was] optimized to make the most of the console hardware capabilities" (Evans, Hagiu, and Schmalensee, 2006, p.130).

Last but not least, our data indicate that in situation of platform overthrow, technical innovations can aim at changing the platform boundaries. For example, case 9 shows how the Palm platform was overthrown because its competitor Handspring provided a technology that disrupted technical core boundaries. At that time, Personal Digital Assistants (PDAs) were Palm's most famous products on which the company relied to fuel its platform business model. Handspring proposed two alternative technologies, Visors and SpringBoard that halted Palm's success because of technical adaptability: "A major disadvantage of the original Palm hardware architecture (especially in contrast to SpringBoard) was the lack of an expansion slot or standard connector that made it easy for third parties to add on modules" (Gawer and Cusumanno, 2002, p.208). The issue was clearly technical as "Unlike Palm, [...], Handspring engineers designed the hardware [...] in a bold move to make modules or peripherals as easy as possible to connect. The expansion modules literally snapped into the expansion slot on the back of the Visor PDA. The Palm device lacked such a simple mechanism for expansion when Handspring introduced this innovation" (Gawer and Cusumanno, 2002, p.208). By proposing a technical core that could cope with functional expansion, Handspring succeeded in challenging Palm's architectural control of the platform: "although Handspring was helping to build broader acceptance of Palm OS as a standard platform, the company seemed to be doing more than Palm to drive forward this PDA architecture". (Gawer and Cusumanno, 2002, p.208)

During an attempt of platform overthrow, players modify their platform cores, either by reconfiguring them, by using a new technology or by changing the technical core boundaries. According to our data, the main objective of these technical core changes seems to be coping with functional expansion. We therefore suggest to analyze these strategic moves as mechanisms that aim at increasing the genericity of the technical core.

The genericity of a technology is a notion that is rooted in the engineering design literature (Le Masson et al. 2017). Generic technologies are defined as technologies that are "able to address many existing and emerging markets and create value across a broad range of applications" (Kokshagina et al. 2016). For the purpose of this study, we slightly adapt this definition and postulate that generic technologies are "able to address many existing and emerging functions and create value across a broad range of applications". Put differently, a generic technology is therefore a technology that can cope with functional expansion. In our view, the genericity is a notion that encapsulates the technical innovations designed during an attempt of platform overthrow. Figure 2 sums up the inductive logic that led us to that conclusion. We illustrate through this figure the analysis aforementioned and account for all the cases in which quotes appear that relate either to technical reconfiguration, technical transition or technical change of boundaries. We also included, when we had enough space, other quotes from different cases in order to better illustrate the notion of genericity.

Insert Figure 2 here

\subsection{Synthesis of the results: a statistical test to strengthen the insights of our inductive protocol}

Our inductive protocol suggests that during an attempt of platform overthrow, both the challenger and the platform leader can engage in functional expansion and try to increase the genericity of their technical core. However, as emphasized in our methodological section, the richness of our empirical material goes beyond the identification of the innovations conducted: it enables to know which player performs which innovation in a given case. Furthermore, the empirical material also provides the outcome of the competitive struggle. We can therefore conduct an analysis that links the following variables: who performed which type of innovation and with which outcome?

The first pattern that emerge from our data is that there is systematically at least one quote in each case that indicates that the challenger performed functional expansion. As mentioned in the methodology section, a case belongs to our sample only if we found an explicit quote that states that the challenger is trying to overthrow the platform. Therefore, by construction, the belonging to the sample is independent to the fact that the challenger performed functional 
expansion. Nevertheless, in our research material, these variables are systematically concomitant. From this observation, we can formulate the following proposition:

Proposition 1: Every attempt of platform overthrow is built on a functional expansion that originates from the challenger

As indicated, the functional expansion can be solving long lasting functional issues faced by the platform leader, adding a new function on top of the existing ones or increasing the potential of the platform ecosystem. The data suggest that established platform leaders can also performed such functional expansion but the striking fact that Proposition 1 accounts for is that all challengers - whether successful or not in overthrowing the platform - conduct functional expansion.

The second pattern that emerges from our data relates to the link between increasing the genericity of a player's technical core and the outcome of the competitive struggle. In our data, we found:

- 15 cases in which the challenger increased the genericity of its technical core and succeeded in its overthrow's attempt (situation 1).

- 12 cases in which the platform leader increased the genericity of its technical core and resisted the attempt of overthrow (situation 2)

- 0 cases in which the platform leader increased the genericity of its technical core and was nevertheless overthrown (situation 3)

- 0 cases in which the challenger increased the genericity of its technical core and failed nevertheless in its overthrow's attempt (situation 4)

Therefore, there seems to be a close relationship between increasing the genericity of a technical core and the outcome of the competitive struggle. We tested a neutral hypothesis that stipulates a uniform distribution among the four situations (situations $1,2,3$ and 4 ) with a $\chi 2$ test with a $0.1 \%$ confidence interval. We obtain a total distance of 27 , which is superior to the table distance of 6.63. Thus, we rejected the neutral hypothesis of a uniform distribution between the genericity variable and the outcome variable and concluded as followed: if a challenger wants to overthrow a platform, it must increase the genericity of its technical core and if a platform leader wants to survive an attempt of platform overthrow, it must increase the genericity of its technical core. From this conclusion, we formulate the following proposition:

Proposition 2: The winner of an attempt of platform overthrow increased the genericity of its technical core

As indicated, a player can increase the genericity of its technical core by recomposing it, by proposing a new technology on which the technical core can be based or by changing the boundaries of its technical core.

\section{5) Discussion: Contributions, limitations and implications for the future of digital platform competition}

\subsection{Contributions to the field of digital platforms}

This article makes several contributions to the field of digital platform.

Our first contribution is the identification of platform overthrow as an important but yet underexplored topic in the platform literature. In this article, we define platform overthrow as a situation in which a platform leader loses its architectural control of the platform for the profit of a challenger that, initially, is strategically weaker. As theoretical models in the literature emphasize platform leader stability, one could have expected platform overthrow to be a rather scarce situation. However, by gathering a sample of 27 descriptions of platform overthrow attempt that were (for the vast majority) academic-related, our article shows that platform overthrow situations are not uncommon. Furthermore, the concept of platform overthrow provides an analytical lens to account for empirical works that describe the fall of platform leaders (Gallagher and Park 2002; Kenney and Pon 2011; West and Wood 2013; Den Hartigh et al. 2016). With the concept of platform overthrow, we contribute to the nascent literature on platform life-cycle (Muzellec, Ronteau, and Lambkin 2015; Teece 2017; Reeves et al. 2019). Indeed, the literature displays a bias toward the early stages of platform life-cycle, at the expense of what happens during platform maturity and platform fall (Gawer 2020). With the concept of platform overthrow, we underline that even efficient platform leaders can fall and therefore we enrich the theoretical representation of both the maturity stage and the fall stage of platform life-cycle. 
Our second contribution relates to the characterization of functional and technical innovations conducted within a platform ecosystem. In this article, we identify that, during an attempt of platform overthrow, both the platform leader and the challenger undertake functional expansion and technical genericity. We conceptualize functional expansion as a functional innovation that is neither an optimization, nor a combination of existing functions. More precisely, we show that a player can conduct functional expansion by solving long lasting functional issues, adding new functions on top of the existing ones and increase the potential of the platform ecosystem. In this article, technical genericity nominates the ability of a technology to address many existing and emerging functions and create value across a broad range of applications. We document that a technical core reconfiguration, a technical core transition and a technical core change of boundaries can be leveraged to increase the technical core's genericity of a platform ecosystem. Scholars that study platforms have for long underlined that such ecosystems foster innovations. Therefore, specific forms of functional expansion and technical genericity have been reported on by the literature. Thus, the work on platform envelopment strategy (Eisenmann, Parker, and Van Alstyne 2011) already showed that adding new functions on top of existing ones can destabilized an established platform leader. The notion of platform leaders' dilemma (Gawer and Cusumano 2014) highlights the importance for a platform leader to solve long-lasting functional issues to prevent competitive threats. Poorly-managed platform core transitions (Ozalp, Cennamo, and Gawer 2018) and poorly-controlled technical core boundaries (Karhu and Ritala 2020) have already been identified as threatening situations for an established platform leader. However, as the literature often conceptualizes platforms as monolithic entities (McIntyre and Srinivasan 2017), few studies provide an holistic view of the innovation dynamics at stake within a mature platform ecosystem (for an interesting exception, see (Gawer 2020)). In particular, to the best of our knowledge, the literature has not yet proposed a structured framework that accounts for the functional and technical innovations orchestrated by mature platform leaders. In our view, the analysis of functional expansion and technical genericity provided in this article is a first step toward such a framework.

Our third contribution is the identification of strategies that can lead to platform overthrow. Fueled by empirical examples, the literature has for long suspected the existence of specific strategies that weaken an established platform leader (Gawer 2014) to the point of dethroning him (Suarez and Kirtley 2012). However, as we demonstrate in the literature review, the literature provides a rather fuzzy description of such strategies, making it difficult for players to implement them. Our results contribute to clarifying these strategies because, by articulating Proposition 1 and Proposition 2, we obtain the following strategic guidelines, formulated from the actors' perspectives:

- Challengers' perspective: to overthrow an established platform, a challenger should introduce a functional expansion based on a generic technical core

- Platform leaders' perspective: to resist an attempt of overthrow, the platform leader should increase the genericity of its technical core in order to cope with the functional expansion brought by the challenger

Our results have clear relevance for managerial practices. First, the notion of platform overthrow has implications for platform managers as it makes them aware that even a weaker player can be a threat. Second, the identified strategies to conduct platform overthrow can be used by platform managers to prioritize their innovation efforts. In particular, our results suggest that both platform and challenger managers should dedicate particular efforts to develop functions that are neither an optimization, nor a combination of existing functions and that they should undertake technical changes that increase the genericity of their platform cores ${ }^{2}$.

\subsection{Limitations and further researches on digital platforms}

This study presents several limitations that call for further research.

First, the limitations are linked with the inductive protocol we follow. In such a protocol, scholars are invited to "focus particular attention on nascent concepts that don't seem to have adequate theoretical referents in the existing literature" (Gioia, Corley, and Hamilton 2012). Indeed, by following this protocol, we identify two concepts, namely functional expansion and technical genericity, that are rooted respectively in industrial economic and in engineering design, but which are loosely related to the main constructs of the platform literature. Therefore, further research is needed to articulate functional expansion and genericity with the main constructs of the platform literature such as platform sides, platform openness, modularity or network effects. In this perspective, several questions remain open such as: Could a platform side be modeled as a bundle of functions? How does platform openness relate to technical genericity? Does modularity always enable technical genericity or are there modular architectures that prevent genericity? Does a

\footnotetext{
2 We can note that our data suggest that platform overthrow outcome cannot be explain without technical genericity. This suggest a slight prevalence of technical issues over market-centric issues in digital platform competitions that manager must be aware of. We thank an anonymous reviewer for this insight
} 
functional expansion always trigger network effects? Answering these questions would enrich the theoretical representation of platform life-cycle.

Second, this article focuses on the notion of platform overthrow that we introduce based on empirical examples. This is a potential bias that limits the generality of our results as we only investigate on one type of platform fall, probably because it is the most easily spotted as it entails an aggressive strategic move from another player. However, there might be a lot of ways for a platform to fall even if the literature has not yet investigated this question (Gawer 2020). Thus, the bourgeoning work on platform fall (Ozalp, Cennamo, and Gawer 2018; Karhu and Ritala 2020) is restricted to one industry and investigates a specific type of platform fall. Therefore, future research could look for a typology of platform falls that is robust throughout different industries.

Third, in this article, we only investigate two mechanisms that lead to platform overthrow, namely functional innovation and technical innovation. However, as previously mentioned, mechanisms such as change of legislation (Bazarhanova, Yli-Huumo, and Smolander 2019), state-supported competitor (Cusumano, Gawer, and Yoffie 2019), worldwide pandemic (Rani and Dhir 2020), lack of trust in the network (Gawer and Henderson 2007), poorly designed pricing system (K. J. Boudreau and Jeppesen 2015) or lack of leadership (Reeves et al. 2019) are all identified by the literature as able to weaken an installed platform leader. Therefore, further research is needed to identify and characterize the different mechanisms that can lead to platform overthrow. Consistently with our previous point, we call for research that articulates the mechanisms that weaken a platform ecosystem with the type of fall a platform leader may be exposed to. Scholars may find that a research protocol based on the interpretations of already published cases, as presented in this paper, can be helpful in providing new insights on such articulation.

Last but not least, by construction, our research is retrospective as we analyze past cases of platform overthrow attempt. Therefore, further research is needed to evaluate the relevance of strategies based on functional expansion and technical genericity and to develop methods and tools that could help managers implementing them.

\subsection{Conclusion: functional expansion, technical genericity and the future of digital platforms}

What do the notions of functional expansion and technical genericity tell us about the future of digital platforms?

On the bright side, functional expansion and technical genericity indicate that current digital platform leaders are not all here to stay (de Reuver, Sørensen, and Basole 2018). As several established platform leaders are attracting the wrath of consumer, complementors and policy makers alike due to their alleged low consideration for social welfare, platform leaders' instability can be seen as good news. Furthermore, functional expansion and technical genericity even give first recipes for challengers that better take into account social and environmental issues to arise.

However, on a less bright side, functional expansion and technical genericity can also tell a totally different story. As several established platform leaders are now more that fifteen years old, one could hypothesizes that they are already able to carefully manage functional expansion and technical genericity. If this guess is true, it could lead to a vicious circle in which a platform leader in a monopolistic position uses functional expansion as a decoy to discourage potential challengers: the monopolistic platform leader constantly introduces new functions to keep challengers at bay. This results in a functional hypertrophy that is neither useful for the consumer, nor aligned with environmental considerations.

From the notions of functional expansion and technical genericity, we therefore foresee two possible futures for digital platform competition. In the first one, consumers, complementors and policy makers succeed in organizing a digital platform competition based on responsible functional expansions (for example by promoting environmentally-related functions). Responsible generic technical cores would then spur and enhance the value platform leaders bring to society. In a second future, aggressive platform leaders confiscated the functional expansion and use it as a way to preserve their monopolistic position. Generic technical cores would then be developed to address unnecessary functions that would decrease value for society. We do hope our work paves the way for researches that will contribute to shape the firstlydescribed desirable future.

\section{6) Bibliography}

Baldwin, Carliss Y, and Kim B Clark. 2006. 'Modularity in the Design of Complex Engineering Systems'. In Complex Engineered Systems: Science Meets Technology, edited by Dan Braha, Ali Minai, and Yaneer Bar-Yam, Springer. New York. doi:10.1007/3-540-32834-3_9.

Baldwin, Carliss Y, and C Jason Woodard. 2007. 'Competition in Modular Clusters'. In Harvard Business School Working Paper, 50.

. 2009. 'The Architecture of Platforms: A Unified View'. In Platforms, Markets and Innovation, edited by 
Annabelle Gawer, 19-44. Cheltenham: Edward Elgar. doi:10.2139/ssrn.1265155.

Bazarhanova, Anar, Jesse Yli-Huumo, and Kari Smolander. 2019. 'From Platform Dominance to Weakened Ownership: How External Regulation Changed Finnish e-Identification’. Electronic Markets, January.

doi:10.1007/s12525-019-00331-4.

Boudreau, Kevin. 2012. 'Let a Thousand Flowers Bloom? An Early Look at Large Numbers of Software "Apps"

Developers and Patterns of Innovation'. Organization Science 23 (5): 1409-27. doi:10.2139/ssrn.1826702.

Boudreau, Kevin J., and Lars B. Jeppesen. 2015. 'Unpaid Crowd Complementors: The Platform Network Effect

Mirage: Unpaid Crowd Complementors'. Strategic Management Journal 36 (12): 1761-77. doi:10.1002/smj.2324.

Bunnell, David, and Adam Brate. 2000. Making the Cisco Connection: The Story behind the Real Internat Superpower. John Wiley \& Sons.

Cennamo, Carmelo, and Juan Santalo. 2013. 'Platform Competition: Strategic Trade-Offs in Platform Markets'.

Strategic Management Journal 34 (11): 1331-50. doi:10.1002/smj.2066.

Cusumano, Michael A., Annabelle Gawer, and David B. Yoffie. 2019. The Business of Platforms: Strategy in the Age of Digital Competition, Innovation, and Power. Edited by HarperBusiness.

Cusumano, Michael A., and Richard W. Selby. 1988. Microsoft Secrets: How the World's Most Powerful Software

Company Creates Technology, Shapes Markets, and Manages Peaople. Edited by Free Press. 1st Touchs.

Den Hartigh, Erik, J. Roland Ortt, Geerten Van de Kaa, and Claire C.M. Stolwijk. 2016. 'Platform Control during

Battles for Market Dominance: The Case of Apple versus IBM in the Early Personal Computer Industry'. Technovation 48-49 (February): 4-12. doi:10.1016/j.technovation.2015.12.001.

Eisenmann, Thomas, Geoffrey Parker, and Marshall Van Alstyne. 2011. 'Platform Envelopment'. Strategic

Management Journal 32 (12): 1270-85. doi:10.1002/smj.935.

El Qaoumi, Kenza, Pascal Le Masson, Benoit Weil, and Aytunç Ün. 2018. 'Testing Evolutionary Theory of Household Consumption Behavior in the Case of Novelty - a Product Characteristics Approach'. Journal of Evolutionary

Economics 28 (2): 437-60. doi:10.1007/s00191-017-0521-9.

Evans, David S. 2009. 'How Catalysts Ignite: The Economics of Platform-Based Start-Ups'. Platforms, Markets and Innovation, no. September 2008: 99-128. doi:10.2139/ssrn.1279631.

Evans, David S., Andrei Hagiu, and Richard Schmalensee. 2006. Invisible Engines How Software Platforms Drive Innovation and Transform Industries. doi:10.1016/0002-9610(46)90380-7.

Fürstenau, Daniel, Carolin Auschra, Stefan Klein, and Martin Gersch. 2019. 'A Process Perspective on Platform Design and Management: Evidence from a Digital Platform in Health Care’. Electronic Markets 29 (4): 581-96.

doi:10.1007/s12525-018-0323-4.

Gallagher, Scott, and Seung Ho Park. 2002. 'Innovation and Competition in Standard-Based Industries: A Historical Analysis of the U.S. Home Video Game Market'. IEEE Transactions on Engineering Management 49 (1). IEEE: 6782. doi:10.1109/17.985749.

Garud, Raghu, and Arun Kumaraswamy. 1993. 'Changing Competitive Dynamics in Network Industries: An Exploration of Sun Microsystems’ Open Systems Strategy’. Strategic Management Journal 14 (5): 351-69. doi:10.1002/smj.4250140504.

Gawer, Annabelle. 2014. 'Bridging Differing Perspectives on Technological Platforms: Toward an Integrative Framework'. Research Policy 43 (7). Elsevier B.V.: 1239-49. doi:10.1016/j.respol.2014.03.006.

. 2020. 'Digital Platforms' Boundaries: The Interplay of Firm Scope, Platform Sides, and Digital Interfaces'. Long Range Planning, September, 102045. doi:10.1016/j.1rp.2020.102045.

Gawer, Annabelle, and Michael A. Cusumano. 2002. Platform Leadership: How Intel, Microsoft and Cisco Drive Industry Innovation. Harvard Business School Press.

. 2014. 'Industry Platforms and Ecosystem Innovation'. Journal of Product Innovation Management 31 (3): 417-33. doi:10.1111/jpim.12105.

Gawer, Annabelle, and Rebecca Henderson. 2007. 'Platform Owner Entry and Innovation in Complementary Markets: Evidence from Intel'. Journal of Economics \& Management Strategy 16 (1): 1-34.

Geissinger, Andrea, Christofer Laurell, and Christian Sandström. 2020. 'Digital Disruption beyond Uber and AirbnbTracking the Long Tail of the Sharing Economy’. Technological Forecasting and Social Change 155 (June): 119323. doi:10.1016/j.techfore.2018.06.012.

Gioia, Dennis A., Kevin G. Corley, and Aimee L. Hamilton. 2012. 'Seeking Qualitative Rigor in Inductive Research: Notes on the Gioia Methodology'. Organizational Research Methods 16 (1): 15-31. doi:10.1177/1094428112452151. Glimstedt, Henrik. 2020. 'The iPhone and its antecedents: re-thinking entry and the evolution of platform strategies at Apple Inc.' Entreprises et histoire n'98 (1): 120. doi:10.3917/eh.098.0120.

Hatchuel, Armand, Pascal Le Masson, and Benoit Weil. 2009. 'Platforms for the Design of Plateforms: Collaborating in the Unknown'. In Platforms, Markets and Innovation, edited by Annabelle Gawer. Edward Elgar.

doi:10.4337/9781849803311.00019.

Hein, Andreas, Maximilian Schreieck, Manuel Wiesche, Markus Böhm, and Helmut Krcmar. 2019. 'The Emergence of Native Multi-Sided Platforms and Their Influence on Incumbents'. Electronic Markets 29 (4): 631-47.

doi:10.1007/s12525-019-00350-1.

Karhu, Kimmo, and Paavo Ritala. 2020. 'Slicing the Cake without Baking It: Opportunistic Platform Entry Strategies in Digital Markets’. Long Range Planning, March, 101988. doi:10.1016/j.lrp.2020.101988.

Kay, Neil M. 2013. 'Rerun the Tape of History and QWERTY Always Wins'. Research Policy 42 (6-7): 1175-85. 
doi:10.1016/j.respol.2013.03.007.

Kazan, Erol, Chee Wee Tan, Eric T.K. Lim, Carsten Sørensen, and Jan Damsgaard. 2018. 'Disentangling Digital Platform Competition: The Case of UK Mobile Payment Platforms'. Journal of Management Information Systems 35 (1): 180-219. doi:10.1080/07421222.2018.1440772.

Keating, Gina. 2013. Netflixed: The Epic Battle for America's Eyeballs. Portfolio.

Kenney, Martin, and Bryan Pon. 2011. 'Structuring the Smartphone Industry: Is the Mobile Internet OS Platform the Key?' Journal of Industry, Competition and Trade 11 (3): 239-61. doi:10.1007/s10842-011-0105-6.

Kokshagina, Olga, Pascal Le Masson, Benoit Weil, and Patrick Cogez. 2016. 'Portfolio Management in Double Unknown Situations: Technological Platforms and the Role of Cross-Application Managers'. Creativity and Innovation Management 25 (2): 270-91. doi:10.1111/caim.12121.

Le Masson, Pascal, Armand Hatchuel, Olga Kokshagina, and Benoit Weil. 2017. 'Designing Techniques for Systemic Impact: Lessons from C-K Theory and Matroid Structures'. Research in Engineering Design 28 (3): $275-98$. doi:10.1007/s00163-016-0241-4.

Legrand, Julien, Maxime Thomas, Pascal Le Masson, and Benoît Weil. 2017. 'Rise and Fall of Platforms: Systematic Analysis of Platform Dynamics Thanks to Axiomatic Design'. Proceedings of the International Conference on Engineering Design, ICED 7: 51-60.

McIntyre, David P., and Arati Srinivasan. 2017. 'Networks, Platforms, and Strategy: Emerging Views and next Steps'. Strategic Management Journal 38 (1): 141-60. doi:10.1002/smj.2596.

Muzellec, Laurent, Sébastien Ronteau, and Mary Lambkin. 2015. 'Two-Sided Internet Platforms: A Business Model Lifecycle Perspective'. Industrial Marketing Management 45 (1): 139-50. doi:10.1016/j.indmarman.2015.02.012. Ozalp, Hakan, Carmelo Cennamo, and Annabelle Gawer. 2018. 'Disruption in Platform-Based Ecosystems'. Journal of Management Studies 55 (7): 1203-41. doi:10.1111/joms.12351.

Parker, Geoffrey G., Marshall W. Van Alstyne, and Sangeet Paul Choudary. 2016. Platform Revolution: How Networked Markets Are Transforming the Economy and How to Make Them Work for You. Edited by WW Norton \& Co.

Rani, Uma, and Rishab Kumar Dhir. 2020. 'Platform Work and the COVID-19 Pandemic'. The Indian Journal of Labour Economics, no. 63: 163-71.

Reeves, Martin, Hen Lotan, Julien Legrand, and Michael G Jacobides. 2019. 'How Business Ecosystems Rise ( and Often Fall )'. MIT Sloan Management Review, no. 1: 1-8.

Reuver, Mark de, Carsten Sørensen, and Rahul C. Basole. 2018. 'The Digital Platform: A Research Agenda'. Journal of Information Technology 33 (2): 124-35. doi:10.1057/s41265-016-0033-3.

Rindova, Violina P, Adrian Yeow, Luis L Martins, and Samer Faraj. 2012. 'Partnering Portfolios, Value-Creation Logics, and Growth Trajectories: A Comparison of Yahoo and Google (195 to 2007)'. Strategic Entrepreneurship Journal, no. 6: 133-51. doi:10.1002/sej.1131.

Rochet, Jean-charles, and Jean Tirole. 2003. 'Platform Competition in Two-Sided Markets'. Journal of the European Economic Association 1 (4): 990-1029. doi:10.1162/154247603322493212.

Sanasi, Silvia, Antonio Ghezzi, Angelo Cavallo, and Andrea Rangone. 2020. 'Making Sense of the Sharing Economy: A Business Model Innovation Perspective'. Technology Analysis \& Strategic Management 32 (8): 895-909. doi:10.1080/09537325.2020.1719058.

Schmidt, Alexander Lennart, Neele Petzold, Norman Lahme-Hütig, and Florian Tiemann. 2020. 'Growing with Others: A Longitudinal Study of an Evolving Multi-sided Disruptive Platform'. Creativity and Innovation Management, August, caim.12401. doi:10.1111/caim.12401.

Staykova, Kalina S., and Jan Damsgaard. 2015. 'The Race to Dominate the Mobile Payments Platform: Entry and Expansion Strategies'. Electronic Commerce Research and Applications 14 (5): 319-30.

doi:10.1016/j.elerap.2015.03.004.

Steinberg, Marc. 2017. 'A Genesis of the Platform Concept: I-Mode and Platform Theory in Japan'. Asiascape: Digital Asia 4 (3): 184-208. doi:10.1163/22142312-12340077.

Suarez, Fernando, and Jacqueline Kirtley. 2012. 'Dethroning an Established Platform'. MITSloan Management Review 53 (4): 9.

Teece, David J. 2017. 'Dynamic Capabilities and (Digital) Platform Lifecycles'. In Advances in Strategic Management, edited by Jeffrey Furman, Annabelle Gawer, Brian S. Silverman, and Scott Stern, 37:211-25. Emerald Publishing Limited. doi:10.1108/S0742-332220170000037008.

Thomas, Llewellyn, Erkko Autio, and David Gann. 2014. 'Architectural Leverage: Putting Platforms in Context.' Academy of Management Perspectives 28 (2): 198-219. doi:10.5465/amp.2011.0105.

West, Joel, and David Wood. 2013. 'Evolving an Open Ecosystem: The Rise and Fall of the Symbian Platform'. In Advances in Strategic Management, edited by Ron Adner, Joanne E. Oxley, and Brian S. Silverman, 30:27-67. Emerald Group Publishing Limited. doi:10.1108/S0742-3322(2013)0000030005.

Yoffie, David B., Annabelle Gawer, and Michael A. Cusumano. 2019. 'A Study of More than 250 Platforms a Reveal Why Most Fail'. Harvard Business Review. 


\section{7) Tables and figures}

Table 1: Sources Contribution Description

\begin{tabular}{|c|c|c|c|}
\hline \multirow{2}{*}{ Source reference } & \multirow{2}{*}{$\begin{array}{l}\text { Number of } \\
\text { cases from } \\
\text { the source }\end{array}$} & \multicolumn{2}{|r|}{ Example of one case from the source } \\
\hline & & $\begin{array}{l}\text { Platform Leader } \\
\text { versus Challenger }\end{array}$ & Platform overthrow attempt quotations \\
\hline $\begin{array}{l}\text { (Annabelle Gawer } \\
\text { and Cusumano 2002) }\end{array}$ & 8 & $\begin{array}{l}\text { Microsoft } \\
\text { vs. } \\
\text { Netscape }\end{array}$ & $\begin{array}{l}\text { "Customers can launch software applications from Web servers that interact with } \\
\text { [Netscape] rather than with Windows, making the browser a competing platform" (p.93) }\end{array}$ \\
\hline $\begin{array}{l}\text { (Evans, Hagiu, and } \\
\text { Schmalensee 2006) }\end{array}$ & 6 & $\begin{array}{l}\text { Microsoft } \\
\text { vs. } \\
\text { IBM }\end{array}$ & $\begin{array}{l}\text { "IBM released OS/2 } 2.0 \text { in } 1992 \text { with the slogan 'a better DOS than DOS, and a better } \\
\text { Windows than Windows", (p.94) }\end{array}$ \\
\hline $\begin{array}{l}\text { (Cusumano, Gawer, } \\
\text { and Yoffie 2019) }\end{array}$ & 3 & $\begin{array}{c}\text { eBay } \\
\text { vs. } \\
\text { Alibaba }\end{array}$ & $\begin{array}{l}\text { "To respond to the challenge posed by eBay, Alibaba took the American firm head-on, } \\
\text { launching a rival C2C marketplace called Taobao" (p.69) }\end{array}$ \\
\hline $\begin{array}{l}\text { (Den Hartigh et al. } \\
\text { 2016) }\end{array}$ & 1 & $\begin{array}{l}\text { Apple } \\
\text { vs. } \\
\text { IBM }\end{array}$ & $\begin{array}{l}\text { "From that moment [Apple II released] onwards, various rival platforms would fight } \\
\text { [IBM] for dominance in the personal computer industry" (p.4) }\end{array}$ \\
\hline $\begin{array}{l}\text { (West and Wood } \\
\text { 2013) }\end{array}$ & 3 & $\begin{array}{l}\text { Microsoft } \\
\text { vs. } \\
\text { Symbian Ltd }\end{array}$ & $\begin{array}{l}\text { "A few months later, Microsoft's CEO Bill Gates termed [Symbian] 'serious } \\
\text { competition"” (p.32) }\end{array}$ \\
\hline (Keating 2013) & 1 & $\begin{array}{c}\text { Netflix } \\
\text { vs. } \\
\text { BlockBuster Online }\end{array}$ & $\begin{array}{l}\text { "[Blockbuster Online CEO said]: they were not Blockbuster crushing a smaller company; } \\
\text { they were a start-up trying to catch a technologically superior and far more experienced } \\
\text { competitor" (p.108) }\end{array}$ \\
\hline
\end{tabular}


Table 2: Sample composition

\begin{tabular}{|c|c|c|c|c|c|c|}
\hline $\mathbf{N}^{\circ}$ & Source Reference & Platform Leader (Tech) versus Challenger (Tech) & $\begin{array}{l}\text { Over } \\
\text { throw }\end{array}$ & Industry & $\begin{array}{c}\text { Theoretical constructs (Modularity/ } \\
\text { Multi-sided) }\end{array}$ & $\begin{array}{c}\text { Platform type } \\
\text { (Innovation/Transaction) }\end{array}$ \\
\hline 1 & \multirow{6}{*}{ Gawer and Cusumano 2002} & IBM (Small Computer System Interface) vs. Intel (USB) & Yes & Personal Computer & Modularity & Innovation \\
\hline 2 & & IBM (The PC Architecture) vs. Intel (PCI) & Yes & Personal Computer & Modularity & Innovation \\
\hline 3 & & IBM (Bios) vs. Microsoft (DOS) & Yes & Personal Computer & Modularity & Innovation \\
\hline 4 & & Microsoft (Internet Explorer) vs. Netscape (Navigator) & Yes & Internet Browser & Modularity & Transaction \\
\hline 5 & & Palm (Palm OS) vs. Handspring (Visors and SpringBoard) & Yes & Electronical devices / Mobiles & Multi-sided & Innovation \\
\hline 6 & & Cisco (Routers) vs. Crescendo (Switches) & No & Internet Infrastructure & Modularity & Innovation \\
\hline 7 & \multirow{2}{*}{$\begin{array}{c}\text { Gawer and Cusumano } 2002 \\
\text { and Bunnell } 2002\end{array}$} & Cisco (Routers) vs. StrataCom (Frame Relay technology) & No & Internet Infrastructure & Modularity & Innovation \\
\hline 8 & & Cisco (IOS) vs. NIA (Open specifications technology) & No & Internet Infrastructure & Modularity & Innovation \\
\hline 9 & \multirow{5}{*}{$\begin{array}{c}\text { Evans, Hagiu and } \\
\text { Schmalensee, } 2006\end{array}$} & Palm (Palm OS) vs. Microsoft (Pocket PC OS) & No & Electronical devices / Mobiles & Multi-sided & Innovation \\
\hline 10 & & Microsoft (Windows 3.0) vs. IBM (OS/2 2.0) & No & Personal Computer & Multi-sided & Innovation \\
\hline 11 & & US video game makers (VCS) vs. Nintendo (NES) & Yes & Video Game & Multi-sided & Innovation \\
\hline 12 & & Nintendo (Super NES) vs. Sony (Playstation) & Yes & Video Game & Multi-sided & Innovation \\
\hline 13 & & Palm (PalmOS) vs. Research in Motion (Blackberry) & Yes & Electronical devices / Mobiles & Multi-sided & Innovation \\
\hline 14 & \multirow{3}{*}{ West and Wood, 2013} & Symbian Ltd. (Symbian OS) vs. Microsoft (Windows CE) & Yes & Electronical devices / Mobiles & Modularity & Innovation \\
\hline 15 & & Symbian Ltd. (Smartphone + OS) vs. Apple (iPhone) & Yes & Electronical devices / Mobiles & Modularity & Hybrid Company \\
\hline 16 & & Symbian Ltd. (Smartphone + OS) vs. Google (Android) & Yes & Electronical devices / Mobiles & Modularity & Hybrid Company \\
\hline 17 & \multirow{3}{*}{$\begin{array}{c}\text { Cusumano, Gawer and } \\
\text { Yoffie, } 2019\end{array}$} & eBay (EachNet) vs. Alibaba (Taobao) & Yes & Online selling & Multi-sided & Transaction \\
\hline 18 & & Microsoft (Internet Explorer) vs. Mozilla (Firefox) & Yes & Internet Browser & Multi-sided & Transaction \\
\hline 19 & & Mozilla (Firefox) vs. Google (Google Chrome) & Yes & Internet Browser & Multi-sided & Transaction \\
\hline 20 & \multirow{2}{*}{ Cusumano and Selby, 1988} & Microsoft (Word) vs. Novell (WordPerfect) & No & Personal Computer & Modularity & Innovation \\
\hline 21 & & Microsoft (Multiplan then Excel) vs. IBM (Lotus) & No & Personal Computer & Modularity & Innovation \\
\hline 22 & \multirow{2}{*}{ Glimstedt, 2020} & Apple (iPod) vs. OEMs (Music Phones) & Yes & Electronical devices / Mobiles & Mixed & Innovation \\
\hline 23 & & OEMs (Music Phones) vs. Apple (iPhone) & Yes & Electronical devices / Mobiles & Mixed & Hybrid Company \\
\hline 24 & Gallagher and Park, 2002 & Atari (Pong-like arcades then VCS) vs. Fairchild (Channel F) & No & Video Game & Mixed & Innovation \\
\hline 25 & Den Hartigh et al, 2016 & IBM (Mainframes then PCs) vs. Apple (Apple II) & No & Personal Computer & Mixed & Innovation \\
\hline 26 & Keating, 2013 & Netflix (DvD then website) vs. (Blockbuster Online) & No & Film industry & Mixed & Transaction \\
\hline 27 & Staykova, Damsgard 2015 & Danske Bank (MobilePay) vs. Banking Sector (Swipp) & No & Banking & Multi-sided & Transaction \\
\hline
\end{tabular}


Figure 1: Data structure concerning the type of functional innovations

\section{Case number 2 : IBM (The PC Architecture) vs. Intel (PCI)}

"We picked (Intel's PCI] because it solved a range of problems: It solved a graphics problem, it solved plug and play problems, it solved interrupt problems, it solved performance issues" (Gawer et Cusumano, 2002, p.27)

\section{Case 18: Microsoft (Internet Explorer) vs. Mozilla (Firefox)}

"Seeking to avoid software bloat and performance isstes, two developers working for the Mozilla nonprofit foundation began what would become the Firefox browser in late 2002. Their goal was to produce a standalone browser that would be faster, simpler, and more secure" (Cusumano, Gawer, Yoffie, 2019, p.72)

\section{Quotes for the "solving" $2^{\text {nd }}$ order theme are also found in cases $6 ; 11 ; 12 ; 19 ; 20 ; 21 ; 23 ; 25$ and 26}

\section{Case number 13 : Palm (PalmOS) vs. Research in Motion (Blackberry)}

"Mozilla introduced its first browser in June 2002, with innovative features such as tabbed browsing, the ability to select a word or phrase and search the web for the selected text, and the ability to store common information for automatically filling in forms" (Cusumano, Gawer, Yoffie, 2019, p.72).

\section{Case number 14 : Microsoft (Windows CE) vs. Symbian Ltd. (Symbian OS)}

"Beyond the ability to make voice calls on GSM mobile phone networks, the phones inherited the capabilities of Psion's organizers (such as a calendar and address book) to which Symbian and its partners added features suitable for a mobile Internet device (such as e-mail and web browsing)" (West and Wood, 2013, p.33)

\section{Quotes for the "adding" $2^{\text {nd }}$ order theme are also found in case $3 ; 5 ; 9 ; 13 ; 14 ; 17 ; 18 ; 19 ; 22 ; 23$ and 25}

\section{Case 17 : eBay (EachNet) vs. Alibaba (Taobao)}

"Taobao's first web pages were copied directly from eBay's design. But Alibaba quickly pivoted, recognizing that one cannot win a platform battle with a me-too product (...) While eBay initially did not let biyers and sellers interact directly (...J. Taobao encouraged bivyers and sellers to communicate with each other and added instant messaging to the website, allowing bryers and sellers to build trust (.... Alibaba launched its own online payment system called Alipay, similar to PayPal, which eBay had acquired shortly before entering the Chinese market. Alipay, unlike PayPal, used an escrow model. At the time of a transaction, funds would go into an escrow account and would only be released after the buyer had received and inspected the item." (Cusumano, Gawer, Yoffie, 2019, p.69-70)

Quotes for the "increasing" $2^{\text {ml }}$ order theme are also found in case $1 ; 4 ; 6 ; 7 ; 8 ; 10 ; 14 ; 15 ; 16 ; 17 ; 23 ; 24$ and 27

2nd Order Themes

Aggregate Dimension

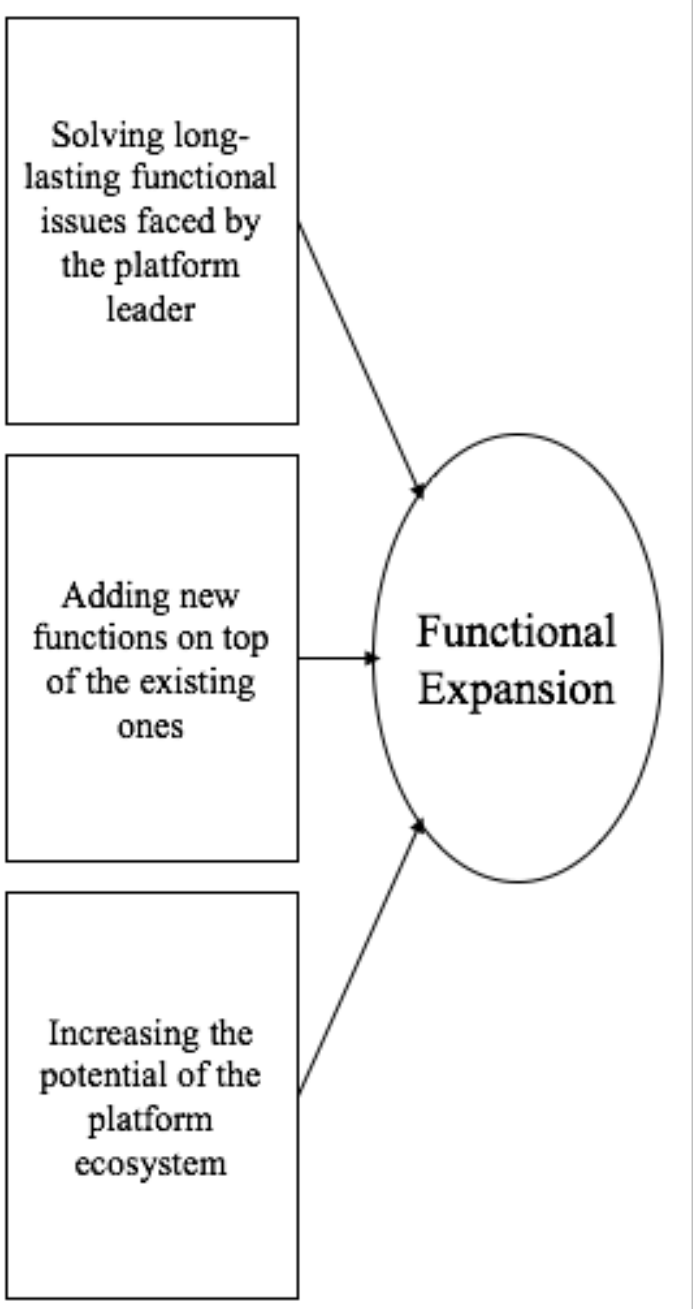


Figure 2 : Data structure concerning the type of technical innovations

\section{Case number 4 : Microsoft (Internet Explorer) vs. Netscape (Navigator)}

"Microsoft executives and senior engineers quickly decided to embed the browser in Windows 95 and head off this threat. /.../

Microsoft (...] built its own browser, Internet Explorer, to add to Windows" (Gawer and Cusumano, 2002, p.145)

"Microsoft took on the challenge [...] bundling a browser with Windows" (Gawer and Cusumano, 2002, p.154).

\section{Case number 7: Cisco (Routers) vs. Crescendo (Switches)}

"bits and pieces of the IOS ran through and connected Cisco technology, (...) One of the first things Cisco did with the technology from acquisitions - StrataCom's switches, for example - was to add IOS code to its platform so that it could work with Cisco's existing product lines" (Bunnel1, 2000, p.118)

\section{Quotes for the "reconfiguration" $2^{\text {nd }}$ order theme are also found in cases $: 3 ; 6 ; 8 ; 12 ; 20 ; 21 ; 27$}

\section{Case 12 : Nintendo (Super NES) vs. Sony (Playstation)}

[Sony] introduced the first commercially successful machine with an operating system and with applications that came on a $C D$ rather than on a chip [...] Nintendo decided to continue using cartridges, both for the Super NES and for the N64 (Evans, Hagiu, and Schmalensee, 2006, p.129-130)

\section{Case number 24 : Atari (Pong-like arcades then VCS) vs. Fairchild (Channel F)}

"[Fairchild's] system used replaceable cartridges, which offered consumers a theoretically inexhaustible variety of games [...] Atari quickly followed with a cartridge-based system of its own, called the Video Computer System " (Gallagher and Park p.71)

Quotes for the "transition" $2^{\text {nd }}$ order theme are also found in cases $10 ; 15 ; 16 ; 22 ; 23 ; 26$

\section{Case number 5 : Palm (Palm OS) vs. Handspring (Visors and SoringBoard) :}

"A major disadvantage of the original Palm hardware architecture (especially in contrast to SpringBoard) was the lack of an expansion slot or standard connector that made it easy for third parties to add on modules [...] Unlike Palm, [...] Handspring engineers designed the hardware [... in a bold move to make modules or peripherals as easy as possible to connect. The expansion modules literally snapped into the expansion slot on the back of the Visor PDA. The Palm device lacked such a simple mechanism for expansion" (Gawer and Cusumanno, 2002, p.208)

Quotes for the "reconfiguration" $2^{\text {nd }}$ order theme are also found in cases $1 ; 2 ; 9 ; 11 ; 13 ; 14 ; 17 ; 18 ; 19 ; 23 ; 25$

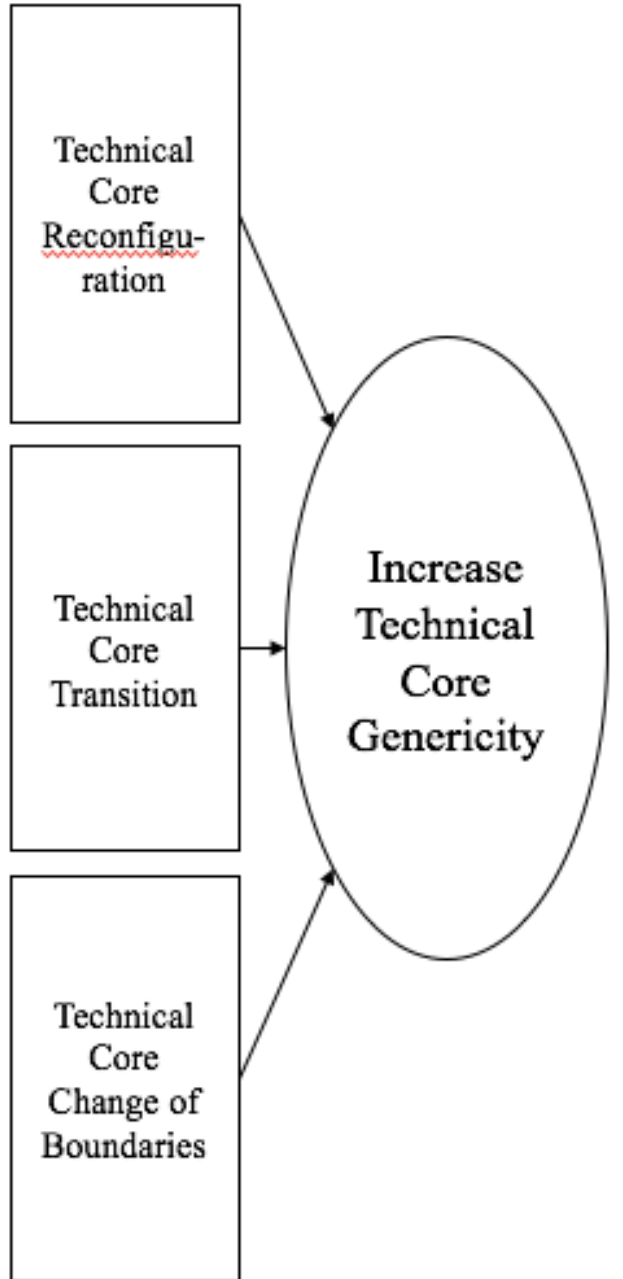

